\title{
Variations on active learning
}

\author{
JOSEPH G. DECK and MARC M. SEBRECHTS \\ Wesleyan University, Middletown, Connecticut
}

\begin{abstract}
Although current theories of memory emphasize the fact that learning is an active process, there is little consensus on the role of active learning. This paper attempts to provide a more precise characterization of active learning in the acquisition of computer skills. The analysis of verbal protocols suggests that active learning varies on at least two dimensions: schema orientation and learning strategy. Schema orientation refers to the type of information the learner is acquiring and varies from relational information to procedural information. Learning strategy describes how the information is acquired. Some people prefer to be internally driven and form their own plans for learning. Others are externally driven and allow the instructional materials to guide their learning. These differences need to be taken into account in describing learning and in designing instructional materials.
\end{abstract}

Research in cognitive science has emphasized the fact that people play an active role in processing information (Gentner \& Stevens, 1983; Miller, Galanter, \& Pribram, 1960). The way objects are perceived, the way another's behavior is analyzed, the way a story is understood are all dependent on schemata (Bartlett, 1932), that is, on some organization of knowledge that the subject has prior to the occurrence of a particular event of interest. These organized structures in memory can be rather well defined for more routine events, like going to a restaurant (e.g., Schank \& Abelson's, 1977, scripts or Minsky's, 1975 , frames); or they can be developed from multiple levels of detail at appropriate times for less routinized behavior (Schank's, 1981, MOPS).

By using such descriptions of memory structures as the basis for any learning task, we can argue that learning is active and subject dependent rather than passive and stimulus dependent. In general, any situation can be dealt with by fitting new information to a prior schema (assimilation) or by changing the schema to fit the new information (accommodation) (Piaget, 1952). Many recent descriptions of learning have emphasized this point. Rumelhart and Norman (1978), for instance, developed a model that emphasizes the fact that learning is based on a continual revision of memorial structure, through processes of accretion, tuning, and restructuring. Accretion consists of the encoding of new information in terms of preexisting schemata. Old schemata are used to interpret new events. These schemata are slowly modified to conform to new situations, through a process of tuning. In some cases, there are no adequate schemata, so new ones are created by a process of restructuring.

This research was supported in part by Grant 82-17572 from the National Science Foundation to the second author. Send correspondence and reprint requests to: Marc M. Sebrechts, Department of Psychology, Wesleyan University, Middletown, CT 06457.

\section{Active Learning of Complex Skills}

We are concerned here with a more detailed description of the active learning of complex skills, specifically, computer skills. Previous work in this area has suggested that apparently simple tasks can turn out to be extremely complex. Bott (1979), for example, found that computer-naive subjects became rather confused about the PRINT command, since they had rather fixed ideas about what printing meant. In one case, a subject interpre ted a PRINT command as indicating that there was a printing press. Likewise, Lewis and Mack (1982) found that temporary office employees often became hopelessly confused when using a word processing system for the first time. Some of the effects could be ascribed to the documentation or system. In other cases, however, there was a mismatch between the strategies the subjects used and the task. Half of their six subjects tried to sign on to the system before they had read how to do so; none were successful. In other cases, subjects drew on prior experiences, which proved misleading. One subject, for example, refused to use a backspace as a way to delete characters, since the backspace did not have the function indicated on the typewriter.

In reviewing some of this work, Carroll and Mack (1982) argued that we have made serious errors in our instructional techniques because we have assumed that the learner is passive. In contrast, they argued, the learner is an active thinker, who structures his or her own learning. Surely, this criticism is largely correct, and the notion of an active learner fits well with the other research mentioned above. However, there do seem to be important differences in the nature of active learning. Three of the Lewis and Mack subjects, for example, did not jump the gun, but were directed by the presented manuals.

There are in fact many meanings attached to the concept "active learning," and this paper provides an analysis of a number of variations in the use of that term. As a context for that analysis, we performed a detailed 
examination of the way in which four people learned to use a new computer system. The results from this limited sample are not meant as a rigid demonstration of categories. Rather, they provide illustrative examples of important conceptual differences that we have ob. served in a number of studies on computer use.

\section{METHOD}

\section{Procedure}

Four people were asked to learn a system that they had not previously used, the Corvus concept. Subjects were presented with all available manuals and told that the task was to become an expert at using the system. No restriction was placed on how long the subjects spent or on how they went about learning how to use the system. They were asked to read aloud material from the screen or the texts and to verbalize their thought processes during the sessions. In order to make sure that subjects learned various aspects of the system, they were also required to complete four tasks in any order they wished: (1) write and run a Pascal program that takes 10 numbers from an input file created by the subject, calculates their mean, and outputs that result to an output file; (2) create a memo; (3) reorganize column data in a document; and (4) revise a long document. The subjects' verbal protocols were recorded, and the execution of their actions was videotaped from the terminal CRT. A few weeks after the completion of training, the four subjects were asked to provide a description of the system in words or diagrams.

\section{Subjects}

The four subjects were experienced computer users but were not primarily computer scientists. Subject experience ranged from one to four operating systems.

\section{System}

The study utilized a Corvus concept. The interface included a series of function keys that had a variable mapping to a set of labels displayed on the bottom of the screen. Most functions could be performed directly through these function keys. The system also had a shell that allowed for direct entry of most commands. The editor was a screen editor that allowed multiple files within a given workspace. Editor functions were also mapped to function keys when the editor was invoked.

\section{RESULTS AND DISCUSSION}

The subjects took between 4 and $10 \mathrm{~h}$ to complete the study. Everyone completed the assigned tasks, but there were differences in how much of the overall structure was learned. Two of the subjects, for example, had virtually no comprehension of the shell at the end of the study. Our primary concern here, however, was to elucidate active learning. An analysis of protocols and subjects' responses to the questions about the system suggested two dimensions on which user learning differed. The first reflected changes to knowledge structures. The second reflected the degree of user control of the learning context. Each of these is considered in turn.

\section{Active Restructuring of Knowledge}

All four subjects in the study formulated a cognitive model (Moran, 1981) of the system based on their prior knowledge. They frequently used analogies (Mayer, 1981 ; Rumelhart \& Norman, 1981) and made comments about how this or that aspect of the system was just like the UCSD P-system, UNIX, or some other system. We could clearly discern a stage that reflected Rumelhart and Norman's accretion through simple statements such as "So this does that" or "This is like the Pascal [P-system] filer." We likewise could see the restructuring described by "Oh, now I get it."

In some cases, the analogies provided speeded acquisition. The two subjects who were familiar with the UCSD P-system, for example, quickly mapped the structure of the Corvus File-Manager onto their knowledge of the structure of the P-system FILER.

In other cases, however, the subjects' prior schemata hindered performance (cf. Halasz \& Moran, 1982; Lewis $\&$ Mack, 1982). The same subjects who successfully used the FILER schemata from the P-system had great difficulty with the Corvus workspace.

Both the UCSD and Corvus systems use the concept of a workspace as a place to do editing; however, beyond this superficial similarity, they have little in common. In the UCSD system, the workspace is a reserved memory location for the current file to be edited and executed. The Corvus workspace, on the other hand, is a specific type of file containing subfiles and directories of subfiles that can be edited, but not directly executed. Based on the similarities, the subjects assumed they knew how the workspace functioned. At first, they were captured by irrelevant aspects of the analogy (Norman, 1981). They quickly discovered that the Corvus workspace was not the same, but it took over $1 \mathrm{~h}$ for them to formulate a correct schema. Both subjects attempted to restructure their schema at first. However, in the end, they abandoned the old schema and imported schema from very different domains. In the case of highly confusable domains, it was apparently easier to abandon the old schema completely and formulate a new schema.

\section{Schema Orientation: Relations or Procedures}

All four subjects followed some pattern of schema retrieval, schema test, schema correction. The nature of the "schema," however, was not the same across subjects. Two of the subjects looked for relational information. They searched for descriptions that would integrate a series of functions into a conceptual unit such as a filer system. They tended to be oriented toward functional aspects of the system. For example, when the manual erroneously described a function key to LIST FILES, one subject inferred that there was a general category of "listing" commands and correctly deduced that the manual should have referred to a LIST VOLUMES function.

The other two subjects were much more oriented toward procedures; they tested their "schemata" against specific commands rather than against general concepts. One subject was so focused on the individual key functions that she referred to what needed to be done in terms of the key numbers rather than in terms of the function. Thus, when she wanted to clear the information on the screen, she said, "I want to see if it will clear this stuff up here. So I have to press F9." She had a good mapping of the key functions, and was the only 
person to notice the mismatch between the spelling of label names in the manual and those on the screen.

These differences were reflected in the descriptions the subjects provided at the end of the study. Two of the subjects concentrated on relations among groups of commands showing overall organization. The other two stressed the specific function keys. Of course, there were variations in the degree to which this was demonstrated. One "relational" subject drew major portions of the system with little regard for specific command names. He indicated the editor, the filer, and the system manager as portions of the system EXEC. As part of the workspace, he listed "I/O" to write and read files; although there is a set of general input and output routines for the Corvus workspace, these represent a general category of functions that are not explicitly labeled as such in the documentation.

In contrast, one of the highly "procedural" subjects described the system in terms of specific function keys. This same person tried to organize the system as relatively modeless, in part reflecting a schema he had of TOPS-20. The other "procedural" subject originally tried to draw a relational diagram, found that to be too complicated, and reverted to listing the command names associated with the function keys.

In summary, there are important differences in the types of prior schemata used to learn new information (Rumelhart \& Norman, 1978). Subjects differ in the extent to which they focus on either procedural or relational information. Procedurally oriented subjects tend to focus on the specific details of particular operations; relationally oriented subjects tend to focus on more global organizational properties.

\section{Learning Strategies: Internally Driven or Externally Driven}

Based on the above description, all of our subjects were active to the extent that they restructured knowledge. However, the way in which this restructuring occurred depended on whether the user relied primarily on prior schemata or on externally provided sources of information.

Two of the subjects made very few references to the manuals; they used their own schemata of the system as a basis for action. Their activity can best be described in terms of goals, plans, and the formulation of specific strategies (Miller et al., 1960). For example, the initial plan of one of these subjects was to see what effects various control characters would have on the system. Next, this subject systematically explored the function keys by guessing what a key would do and then pressing the key to see if his guess was correct. When the subject got lost in the system while following this plan, his strategy was to reboot the system and see if he could replicate his actions. When asked about this action, he said, "It's like playing adventure-when you get hopelessly lost, you kill yourself and start over."

The other two subjects tended to follow the manuals rather closely, although one subject used trial and error after he had finished reading the available materials. The subject who used the manuals throughout the study followed the examples verbatim. This often led to failure, since the examples were meant only as general guides. For instance, after following the example on how to list files and failing to obtain the results in the manual, she made the following comments: "Well, that's not the way it says it should work. I've missed something somewhere." This person tended to structure the task in terms of the manuals rather than in terms of her own prior knowledge. Although there was an error in the manual, she assumed that if there was a mismatch between her model and the one in the manual, then she must be mistaken.

It is important to remember that both internally and externally driven subjects employ active learning insofar as they are developing and revising their schemata. They are not all active, however, in the sense of actively structuring and ordering their own learning. Some subjects actively develop their own plans, and use their prior schemata as the basis for originating new learning. Others rely on an external context to structure changes in their system knowledge. This strategic difference is extremely important, because internally driven (active) learners will require different types of instruction despite the fact that both groups are "actively" restructuring knowledge.

\section{CONCLUSION}

The following conclusions may be made:

(1) Learning necessarily involves active processing. What a person learns is a function of continual structuring and restructuring based on prior knowledge.

(2) There are many possible types of active learning. Two important components that reflect substantial individual differences are the schema orientation used in processing and the learning strategies used to acquire new information.

(3) Active processing results in changing mental representations. These "schemata," however, do not necessarily imply a simple unified structure. Rather, schemata can vary in their specificity. Some people will be relationally oriented and will as a consequence look for overall relations and functions. Others are procedurally oriented; their "schemata" are developed and tested on the basis of more specific procedures. The underlying representation can be thought of as varying from heavily "integrated" to heavily "segregated" (Hayes-Roth, 1977).

(a) Instructional materials frequently emphasize only one of these orientations. As a consequence, the notion of "schemata" alone fails to give sufficient weight to variations in the specific character of those schemata for individual users. One approach is to give more attention to ways of combining relational and procedural information (e.g., Sebrechts, Deck, \& Black, 1984).

(b) Much of the work of restructuring is done by analogy with prior knowledge. In the case of computer 
skills, some analogies are helpful; others actually interfere with understanding. "Near" analogies based on highly similar domains can facilitate quick learning of certain basics, but may make it difficult for people to separate the target domain and the prior schema. "Far" analogies, on the other hand, provide less similarity in procedural details, since they borrow from domains that are dissimilar in many respects; as a consequence, they may be less prone to capture error. (That is, although it may be easier to describe an operating system by reference to another similar operating system, that may lead to far more confusion than using an analogy to a noncomputer office task. The choice, of course, depends on the specific learning objective.)

(4) People differ in the extent to which they actively determine strategies for learning. Some people seem to be externally driven and prefer to follow the sequence of information given. In some cases, they may lack good strategies for organizing the search for new material. It is particularly important to have carefully structured materials for this group. Other people are internally driven. They use prior knowledge as the basis for directing learning. They show less interest in external aids, such as manuals, and prefer trial and error.

(a) In order to accommodate these two types of learning, we need to make available materials in different formats for different people. Some will benefit from tutorials. Others will tend to ignore tutorials and will need reference materials. Of course, this dichotomy is a simplification; there are, in fact, many more subtle differences in user strategies. The extreme categories suggest a first step that can be taken to improve training. Ideally, we would like to design an interactive training system that will be able to adapt to a wide range of learning styles.

\section{REFERENCES}

BARTLETT, F. C. (1932), Remembering: A study in experimental and social psychology. New York: Campbridge University Press. Botr, R. A. (1979). A study of computer learning: Theory and methodologies (Tech. Rep. No. 82). San Diego: University of California, Center for Human Information Processing.

Carroll, J. M., \& Mack, R. L. (1982). Learning to use a word processor: By doing, by thinking, and by knowing (Tech. Rep. No. RC 9481). Yorktown Heights, NY: IBM Watson Research Center.

Gentner, D., \& Stevens, A. L. (1983). Mental models. Hillsdale, NJ: Erlbaum.

Halasz, F., \& Moran, T. (1982). Analogy considered harmful. Proceedings of the Conference on Human Factors in Computer Systems (pp. 383-386). New York: Association for Computing Machinery.

Hayes-Roth, B. (1977). Evolution of cognitive structures and processes. Psychological Review, 84, 260-278.

LEWIS, C., \& MACK, R. (1982). Learning to use a text-processing system: Evidence from "thinking aloud" protocols (pp. 387392). Proceedings of the Conference on Human Factors in Computer Systems. New York: Association for Computing Machinery.

MAYER, R. E. (1981). The psychology of how novices learn computer programming. Computing Surveys, 13, 121-141.

Millen, G., Galanter, E., \& Pribram, K. (1960). Plans and the structure of behavior. New York: Holt-Dryden.

Minsxy, M. (1975). A framework for representing knowledge. In P. H. Winston (Ed.), The psychology of computer vision. New York: McOraw-Hill.

Moran, T. P. (1981). The command language grammar: A representation for the user interface of interactive computer systems. International Journal of Man-Machine Studies, 15, 3-50.

Norman, D. A. (1981). Categorization of action slips. Psychological Review, 88, 1-15.

Piaget, J. (1952). The origin of intelligence in children. New York: International University Press.

Rumelhart, D. E., \& Nonyan, D. A. (1978). Accretion, tuning and restructuring: Three modes of learning. In J. W. Cotten \& R. Klatzky (Eds.), Semantic factors in cognition. Hillsdale, NJ: Erlbaum.

Rumelhakt, D. E., \& Nomyan, D. A. (1981). Analogical processes in learning. In J. R. Anderson (Ed.), Cognitive skills and their acquisition. Hillsdale, NJ: Erlbaum.

Schank, R. C. (1981). Language and memory. In D. A. Norman (Ed.), Perspectives on cognitive science (pp. 105-146). Norwood, NJ: Ablex.

Schanx, R. C., \& Abelson, R. P. (1977). Scripts, plans, goals and understanding. Hillsdale, NJ: Erlbaum.

Sebrechts, M. M., Deck, J. G., \& Black, J. B. (1984). A diagrammatic approach to computer instruction for the naive user. Behavior Research Methods, Instruments, \& Computers, 15, 200-207. 